\title{
Placement of Robot Manipulators to Maximize Dexterity
}

\author{
Karim Abdel-Malek and Wei Yu \\ Department of Mechanical Engineering \\ The University of Iowa \\ Iowa City, IA 52242 \\ Tel. (319) 335-5676 \\ amalek@engineering.uiowa.edu
}

Placement of robotics manipulators involves the specification of the position and orientation of the base with respect to a predefined work environment. A general approach to the placement of manipulators based on the kinematic dexterity of mechanisms is presented. In many robotic implementations, it is necessary to carefully plan the layout of the workplace, whether on the manufacturing floor or in robot-assisted surgical interventions, whereby it is required to locate the robot base in such a way to maximize dexterity at or around given targets. In this paper, we pose the problem in an optimization form without the utilization of an inverse kinematics algorithm, but rather by employing a dexterity measure. A new dexterous performance measure is developed and used to characterize a formulation for moving the workspace envelope (and hence the robot base) to a new position and orientation. Using this dexterous measure, numerical techniques for placement of the robot and based on a method for determining the exact boundary to the workspace are presented and implemented in computer code. Examples are given to illustrate the techniques developed using planar and spatial serial manipulators.

Keywords: Manipulator placement, dexterity measure, reachability, manipulability. 


\section{Introduction}

Manipulator placement in an environment such that it will perform a number of tasks with maximum dexterity is a challenging problem. In this paper, a mathematical formulation is presented based on the kinematics of the manipulator and that does not require obtaining solutions to the inverse kinematics problem.

There has been limited works addressing the placement problem. This is due to the overwhelming focus given to the placing of path trajectories in a robotic environment versus placing the robot in a pre-specified environment. In many cases, the targets cannot be displaced because of physical constraints such as weight or geometry, or because of inability in the case of robotic medical interventions where it is not recommended to move the patient. Early works addressing placement for avoiding interference between the manipulator links was reported by Pamanes-Garcia (1989) and Zegloul and Pamanes-Garcia (1993), while arm reachability as the basic criterion for placement was reported by Seraji (1995).

Workspace volume (Park and Brockett 1994), reachability, and manipulability are measures that have been used in the past (Bergerman and $\mathrm{Xu}$ 1997). Even though the manipulability ellipsoid approach is the most widely used techniques, it has been shown that the manipulability ellipsoid does not transform the exact joint velocity constraints into task space and so may fail to give exact dexterousness measure and optimal direction

of motion in task space. Other types of dexterity measure were proposed by Youheng and Kaidong (1993) and called Average Service Coefficient (ASC) and the Dexterity 
Effective Coefficient (DEC). The authors demonstrated that by deducing the relation formulas between the dexterity indexes and the linkage parameters of manipulators, a dimensional optimum synthesis model can be obtained. Dexterous workspaces have also been addressed by researchers (Wang and Wu 1992 and Yang and Haug 1991) but offers only a general guidance for placement.

A method that does not use inverse kienematics was proposed by Rastegar and Singh (1994) using a probabilistic approach for optimal positioning of task spaces within the workspace of a manipulator.

A mathematical theory for optimizing the kinematic dexterity of robotic mechanisms was presented by Park and Brockett (1994). Using methods from Differential Geometry, this approach takes into account the geometric and topological structures of the joint and workspaces.

Dexterity measures based on the notions of the scaling laws of biological systems were proposed by Sturges (1990). Values for the index of difficulty are shown to vary in the work space, and the loci of maximum dexterity that indicate the most favorable task/effector arrangements are determined.

Kim and Khosla (1991) introduced a number of dexterity measures. For example, the measure of manipulability has an analytical expression, but it depends on the scale of a manipulator. On the other hand, the condition number is independent of the scale, but 
cannot be expressed analytically. These two main problems (scale dependency and analytical expression) of previous dexterity measures were later addresses and applied in the design and control of manipulators.

Manipulability polytopes as a dexterity measure were introduced by Lee (1997) and were compared with manipulability ellipsoids. Extending the concept of manipulability ellipsoids to cooperating arms have been proposed and demonstrated by Bicchi and Melchiorri (1993).

In this paper, we propose a numerical method for the placement of robot manipulators based on maximizing the dexterity at specified target points. We will first define a number of necessary constraints in order to impel the workspace towards target points. We will then define a cost function that is based on a new measure of dexterity at each target point. The problem is then characterized in terms of a maximization function and is addressed using numerical techniques.

\section{Criteria for Impelling the Workspace}

Generalized coordinates $\mathbf{q} \in \mathbf{R}^{n}$ defined as the set of all $n$-tuples $\mathbf{q}=\left[\begin{array}{lll}q_{1} & \cdots & q_{n}\end{array}\right]^{T}$ of real numbers that represent joint variables, where $n$ is the number of degrees of freedom (DOF) of the manipulator. Using the Denavit-Hartenberg representation method (Denavit and Hartenberg 1955), a position vector $\mathbf{x}=\left[\begin{array}{lll}x & y & z\end{array}\right]^{T}$ can be written as

$$
\mathbf{x}=\boldsymbol{\Phi}(\mathbf{q})
$$


where $\boldsymbol{\Phi}: \mathbf{R}^{n} \rightarrow \mathbf{R}^{3}$ is a smooth vector function defined as a subset of the Euclidean space. Surface patches on the boundary of the workspace were delineated in previous work (Abdel-Malek, et al. 1997 and Abdel-Malek, et al. 1999) and is summarized in Appendix A. The result of this work is exact surface patches denoted by $\boldsymbol{\xi}^{(i)} ; i=1, \ldots, m$ in closed form that characterize the boundary of the workspace, including voids if any exist.

In this work, we will consider a number of target points denoted by $\mathbf{p}^{(j)} ; j=1, \ldots, \ell$ that must be not only reached by the end-effector but with maximum dexterity (i.e., can be reached with the maximum number of orientations). To ascertain that target points $\mathbf{p}^{(j)}$ are inside the workspace, additional conditions are needed. The absolute value of the distance between a target point and the boundary should be greater than a specified tolerance denoted by $\varepsilon$. This will guarantee that the target points are located inside of the workspace but not on boundary. The distance between all target points $\mathbf{p}^{(j)}$ and all surface patches $\xi^{(i)}\left(\mathbf{u}^{(i)}\right)$ should be greater than a specified minimum value such as

$$
\left\|\mathbf{p}^{(j)}-\Psi^{(i)}\left(\mathbf{u}^{(i)}, \mathbf{w}\right)\right\| \geq \varepsilon_{j} \text { where } j=1, \ldots, \ell \text { and } i=1, \ldots, m
$$

where we have used a rotation matrix $\mathbf{R}$ to capture the orientation and have used a position vector $\mathbf{v}$ to capture the position of each surface such that the generalized surface is now denoted by $\Psi^{(i)}$ and defined as

$$
\Psi^{(i)}(\mathbf{w}, \mathbf{u})=\mathbf{R}(\alpha, \beta, \gamma) \cdot \boldsymbol{\xi}^{(i)}(\mathbf{u})+\mathbf{v}\left(x_{w}, y_{w}, z_{w}\right)
$$

where the six generalized variables in $\mathbf{w}$ are used to track the workspace envelope.

$$
\mathbf{w}=\left[\begin{array}{llllll}
x_{w} & y_{w} & z_{w} & \alpha & \beta & \gamma
\end{array}\right]^{T}
$$


and where $\varepsilon_{j}>0$ are specified constants. If a target point satisfies both conditions of Eq. (1) and (2), then this point is internal to the workspace (i.e, have placed the workspace in a configuration such that all points are covered by the workspace).

In order to impel the workspace towards the target points, we propose the following two constraints: (1) The target points must satisfy the constraint equation (i.e., located in the workspace volume) and (2) The distance between the target-points and the workspace must satisfy specified constraints in order for the target points not to be located on the boundary. These constraints are defined as follows:

(1) Workspace envelope at least covering the target points (shortest distance between target points):

$$
g_{i} \equiv \min \left\|\mathbf{p}^{(i)}-\Gamma(\mathbf{q}, \mathbf{w})\right\| \leq \varepsilon \quad \text { for } i=1, \ldots, \ell
$$

where the totality of points in the workspace are also translating and rotation in space

$$
\Gamma(\mathbf{w}, \mathbf{q})=\mathbf{R}(\alpha, \beta, \gamma) \Phi(\mathbf{q})+\mathbf{v}\left(x_{w}, y_{w}, z_{w}\right)
$$

and $\beta$ is a very small positive number and subject to the inequality constraints on joint limits as

$$
q_{k}^{L} \leq q_{k} \leq q_{k}^{U} \text { for } k=1, \ldots, n
$$

(2) Embedding the target points inside the workspace volume (a minimum distance between target points and surface patches).

$$
\begin{aligned}
& g_{k} \equiv\left\|\mathbf{p}^{(j)}-\Psi^{(i)}(\mathbf{u}, \mathbf{w})\right\| \geq \varepsilon_{j} \quad \text { for } i=1, \ldots, m \text { and } \\
& \quad j=1, \ldots, \ell, k=1, \ldots,(\ell \times m)
\end{aligned}
$$

where $\varepsilon_{j}$ is the depth of the target point inside the workspace volume. There are $\ell+(\ell \times m)+n$ total number of constraints. 


\section{Placement of the Manipulator for Maximum Dexterity}

In this section, we define a cost function that is based on maximizing the dexterity at target points. Indeed, to mathematically formulate this problem, it is necessary to use an analytic dexterity measure at specific target points that can be manipulated. Because dexterity measures in the literature do not account for joint limits and because of the need for an analytical expression that can be used in the proposed optimization method, we define a new dexterity measure.

\section{Dexterity Measure}

In serial manipulators, joints are constrained, sometimes due to space limitation, others strictly by design and are usually specified by an inequality constraint of the form $q_{i}^{L} \leq q_{i} \leq q_{i}^{U}$, where $q_{i}^{L}$ is the lower limit and $q_{i}^{U}$ is the upper limit. In order to include joint limits in the formulation, we have used a parameterization to convert inequalities on $q_{i}$ to equalities $q_{i}=q_{i}\left(\lambda_{i}\right)$. Joint limits imposed in terms of inequality constraints in the form of $q_{i}^{L} \leq q_{i} \leq q_{i}^{U}$, where $i=1, \ldots n$, are transformed into equations by introducing a new set of generalized coordinates $\lambda=\left[\lambda_{1}, \lambda_{2}, \ldots, \lambda_{n}\right]^{T}$ such that

$$
q_{i}=\left(\left(q_{i}^{L}+q_{i}^{U}\right) / 2\right)+\left(\left(q_{i}^{U}-q_{i}^{L}\right) / 2\right) \sin \lambda_{i} \quad i=1, \ldots, n
$$

These generalized coordinates $\lambda_{i}$ are called slack variables in the field of optimization, where $\lambda=\left[\lambda_{1}, \lambda_{2}, \ldots, \lambda_{n}\right]^{T} \in \mathbf{R}^{n}$. For any admissible configuration $\mathbf{x}_{o}$ and $\mathbf{q}_{o}$, i.e., at a target in space, the following $(n+3)$ augmented constraint equations must be satisfied 


$$
\mathbf{G}(\mathbf{z})=\left[\begin{array}{l}
\boldsymbol{\Phi}(\mathbf{q})-\mathbf{x}_{o} \\
\mathbf{q}(\lambda)-\mathbf{q}_{o}
\end{array}\right]_{(n+3) \times 1}=\mathbf{0}
$$

where the augmented vector of generalized coordinates is $\mathbf{z}=\left[\begin{array}{lll}\mathbf{x}^{T} & \mathbf{q}^{T} & \lambda^{T}\end{array}\right]^{T}$. By defining a new vector $\mathbf{z}^{*}=\left[\begin{array}{ll}\mathbf{q}^{T} & \lambda^{T}\end{array}\right]^{T}$ (input parameters), the augmented coordinates can be partitioned as

$$
\mathbf{z}=\left[\begin{array}{ll}
\mathbf{x}^{T} & \mathbf{z}^{* T}
\end{array}\right]^{T}
$$

The set defined by $\mathbf{G}\left(\mathbf{z}^{*}\right)$ is the totality of points in the workspace that can be touched by the end-effector of a serial robot manipulator while considering joint limits. The input Jacobian of $\mathbf{G}\left(\mathbf{z}^{*}\right)$ is obtained by differentiating $\mathbf{G}$ with respect to $\mathbf{z}^{*}$ as

$$
\mathbf{G}_{\mathbf{z}^{*}}=\left[\begin{array}{cc}
\Phi_{\mathbf{q}} & \mathbf{0} \\
\mathbf{I} & \mathbf{q}_{\lambda}
\end{array}\right]
$$

which is an $(n+3) \times(2 n)$ matrix, where $\Phi_{\mathbf{q}}=\partial \Phi_{i} / \partial q_{j}$ is a $(3 \times n)$ matrix, $\mathbf{I}$ is the $(n \times n)$ identify matrix, and $\mathbf{q}_{\lambda}=\partial q_{i} / \partial \lambda_{j}$ is an $(n \times n)$ diagonal matrix with diagonal elements as $\left.q_{\lambda}\right)_{i i}=b_{i} \cos \lambda_{i}$. We define $\mathbf{G}_{\mathbf{z}^{*}}$ as the augmented Jacobian matrix.

Since this Jacobian inherently combines information about the position, orientation, and joint limits of the end-effector, it is a viable measure of dexterity. Furthermore, because of the simplicity in determining an analytical expression of $\mathbf{G}_{\mathbf{z}}$, it is by far a simpler approach in comparison with the widely used manipulability ellipsoid. We define the dexterity measure as

$$
\mathrm{W}_{d}=\sqrt{\left|\mathbf{G}_{\mathbf{z}^{*}} \mathbf{G}_{\mathbf{z}^{*}}{ }^{T}\right|}
$$


Note that the measure characterized by Eq. (11) takes into consideration all joint limits and singular orientations.

\section{Geometric Significance of the New Dexterity Measure}

1. Relationship with the singular values

A singular value decomposition of the augmented Jacobian matrix can be represented by:

$$
\mathbf{G}_{\mathbf{z}^{*}}(\mathbf{q})=\mathbf{U}_{1} * \Sigma_{1} * \mathbf{V}_{1}^{T}
$$

where $\mathbf{U}_{1} \in \mathbf{R}^{(m+n) \times(m+n)}$ and $\mathbf{V}_{1} \in \mathbf{R}^{2 n \times 2 n}$ are orthogonal matrices, and

$$
\Sigma_{1}=\left[\begin{array}{ccccc}
\sigma_{1} & 0 & \ldots & 0 & 0 \\
0 & \sigma_{2} & 0 & 0 & 0 \\
: & : & \ldots & : & : \\
0 & 0 & \ldots & \sigma_{m+n} & 0
\end{array}\right] \in \mathbf{R}^{(m+n) \times(m+n)}
$$

where $\sigma_{i}, i=1, \ldots, m+n$ are the singular values of $\mathbf{G}_{\mathbf{z}^{*}}$. Substituting Eq.(12) into

Eq.(11) yields

$$
\mathrm{W}_{d}=\sqrt{\operatorname{det}\left(\mathbf{G}_{\mathbf{z}^{*}} \mathbf{G}_{\mathbf{z}^{*}}{ }^{T}\right)}=\sqrt{\operatorname{det}\left(\Sigma_{1} \Sigma_{1}{ }^{T}\right)}
$$

where

$$
\mathrm{W}_{d}=\sigma_{1} * \sigma_{2} * \ldots * \sigma_{m+n}
$$

Equation (15) indicates that the dexterity measure is the product of augmented singular values. When the augmented Jacobian matrix degenerates, one or more singular values will be zeros, then the measure is zero, i.e, some of three type singularities occurs.

\section{Equivalent Ellipsoid Geometry}

From Eq. (10), we have:

$$
\left[\begin{array}{l}
\delta \mathbf{x} \\
\delta \mathbf{q}
\end{array}\right]=G_{\mathbf{z}}\left[\begin{array}{l}
\delta \mathbf{q} \\
\delta \boldsymbol{\lambda}
\end{array}\right] \quad \text { or } \quad \delta \mathbf{R}=G_{\mathbf{z}^{*}} \cdot \delta \mathbf{Q}
$$

which is equivalent to 


$$
\delta \mathbf{R}^{*}=\Sigma_{1} * \delta \mathbf{Q}^{*}
$$

where $\mathbf{R}$ and $\mathbf{Q}$ are called the augmented output vector and augmented input vector, respectively, and

$$
\begin{aligned}
& \delta \mathbf{R}^{*}=\mathbf{U}_{1}{ }^{T} \delta \mathbf{R} \\
& \delta \mathbf{Q}^{*}=\mathbf{V}_{1}^{T} \delta \mathbf{Q}
\end{aligned}
$$

From Eq. (18), we have

$$
\delta R_{i}^{*}=\sigma_{i} \delta Q_{i}^{*} \quad i=1, \ldots, m
$$

When $\delta \mathbf{Q}^{*}$ is constrained by $\left\|\delta \mathbf{Q}^{*}\right\| \leq 1$, then $\delta \mathbf{R}^{*}$ satisfies the following inequality

$$
\frac{\delta R_{1}^{2}}{\sigma_{1}^{2}}+\frac{\delta R_{2}^{2}}{\sigma_{2}^{2}}+\frac{\delta R_{3}^{2}}{\sigma_{3}^{2}}+\cdots+\frac{\delta R_{m+n}^{2}}{\sigma_{m+n}^{2}} \leq 1
$$

This redefined dexterity ellipsoid is of higher dimension as to the dexterity ellipsoid given by $\sqrt{\left|\mathrm{JJ}^{\mathrm{T}}\right|}$. Its volume is computed from

$$
\text { volume }=\frac{\pi^{(m+n) / 2}}{\Gamma(1+(m+n) / 2)} \prod_{i=1}^{m+n} \sigma_{i}
$$

From the above discussion, we draw the following conclusions:

(1) The proposed dexterity measure is more accurate in describing the manipulability of robot than that proposed by Yoshikawa (1995), because it considers all singularities (Jacobian and others) as well as joint limits.

(2) The proposed dexterity measure and that proposed by Yoshikawa (1995) can be interpreted in the same manner in terms of singular values and ellipsoid geometry, but are not directly related. 


\section{Mathematical Modeling of the Placement Problem}

Given $\ell$ target points $\mathbf{P}^{(i)}\left(x_{i}, y_{i}, z_{i}\right)$ for $i=1,2, \cdots, \ell$ defined in space, we introduce a dexterity measure at each point denoted by $W_{d}\left(\mathbf{P}^{(i)}\right)$, where it is necessary to place the manipulator to achieve maximum dexterity at each target point. Note, however, that this is a multi-objective optimization problem. Therefore, we will give dexterity at each point a weight so as to transfer the problem into a usual optimization problem that can be readily solved.

A mathematical model of the placement of the robot base subject to maximizing the dexterity at specified target points is characterized by the following optimization problem.

\section{Cost function}

$$
\operatorname{Maximize} f(\mathbf{w})=\sum_{i=1}^{\ell} \omega_{i} W_{d}\left(\mathbf{w}, \mathbf{p}^{(i)}\right)
$$

where $\omega_{i}, i=1,2, \cdots, \ell$ are specified weights at each target point.

\section{Subject to:}

(1) Target points are inside the workspace volume (Eq. 2).

(2) Target points are not on the boundary of the workspace envelope (Eq. 4).

(3) The manipulator is constrained

$$
\mathbf{q}^{L} \leq \mathbf{q} \leq \mathbf{q}^{U}
$$

(4) The motion is within a finite space

$$
\mathbf{w}^{L} \leq \mathbf{w} \leq \mathbf{w}^{U}
$$


The set of constraints in Eq. (23) is to impose the unilateral constraints on each joint and the second set (Eq. 24) to constrain the motion of the base within a finite space.

The algorithm for achieving placement is shown in Fig. 1.

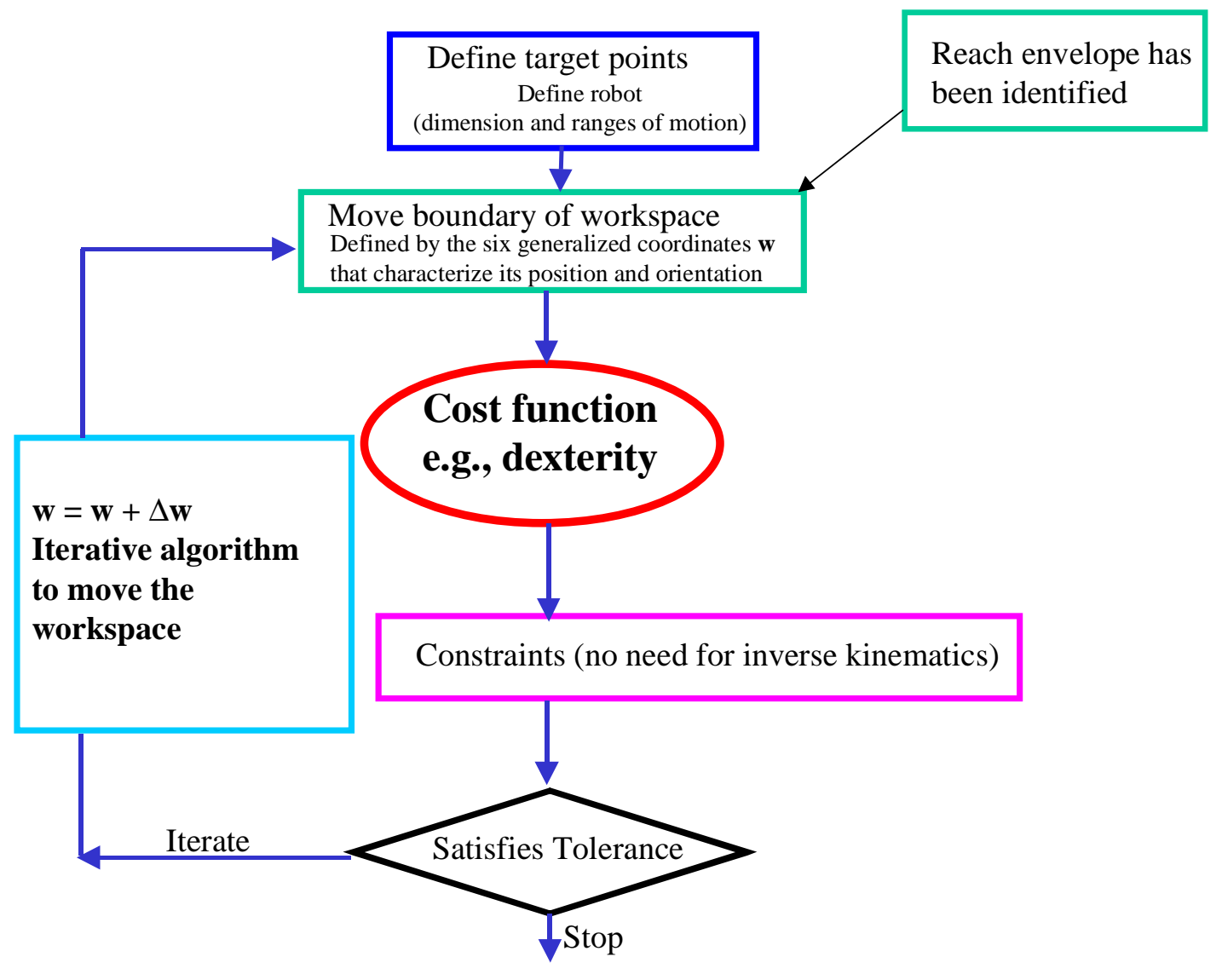

Fig. 1 Algorithm for placement based on optimization

\section{Simple Example}

Consider a planar 3DOF manipulator arm as shown in Fig. 2 consisting of 3 revolute joints. This example will be used to illustrate the theory and will be followed by a more realistic manipulator model of the upper extremity. There are three target points, namely, 
$\mathbf{P}^{(1)}=\left[\begin{array}{ll}14 & 10\end{array}\right]^{T}, \mathbf{P}^{(2)}=\left[\begin{array}{lll}125 & 100 & 50\end{array}\right]^{T}$, and $\mathbf{P}^{(1)}=\left[\begin{array}{lll}125 & 125 & 75\end{array}\right]^{T}$, which must be touched by the end-effector with the ability to reach these points from many directions (i.e., maximizing dexterity).

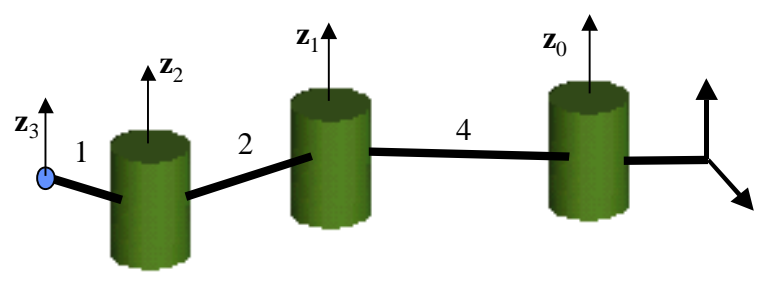

Fig. 2 Model of the arm with three revolute joints

The coordinates of a point on the end-effector are given by

$$
\Phi(\mathbf{q})=\left[\begin{array}{c}
4 \cos q_{1}+2 \cos \left(q_{1}+q_{2}\right)+\cos \left(q_{1}+q_{2}+q_{3}\right) \\
4 \sin q_{1}+2 \sin \left(q_{1}+q_{2}\right)+\sin \left(q_{1}+q_{2}+q_{3}\right)
\end{array}\right]
$$

For simplicity, ranges of motion on each joint are defined as $-\pi / 3 \leq q_{i} \leq \pi / 3 ; i=1,2,3$.

The Jacobian is calculated as

$\Phi_{\mathbf{q}}=\left[\begin{array}{ccc}-4 \sin q_{1}-2 \sin \left(q_{1}+q_{2}\right)-\sin \left(q_{1}+q_{2}+q_{3}\right) & -2 \sin \left(q_{1}+q_{2}\right)-\sin \left(q_{1}+q_{2}+q_{3}\right) & -\sin \left(q_{1}+q_{2}+q_{3}\right) \\ 4 \cos q_{1}+2 \cos \left(q_{1}+q_{2}\right)+\cos \left(q_{1}+q_{2}+q_{3}\right) & 2 \cos \left(q_{1}+q_{2}\right)+\cos \left(q_{1}+q_{2}+q_{3}\right) & \cos \left(q_{1}+q_{2}+q_{3}\right)\end{array}\right]$

Results of the workspace determination yield the following boundary curves (note that curves are generated because we have restricted the manipulator to planar movement. The boundary curves are defined by the following sets:

$$
\begin{array}{ll}
\mathbf{x}\left(\pi / 3, q_{2}, 0\right) ; q_{2} \in[-\pi / 3,0], & \mathbf{x}\left(\pi / 3, q_{2}, 0\right) ; q_{2} \in[0, \pi / 3], \\
\mathbf{x}\left(q_{1}, 0,0\right) ; q_{2} \in[-\pi / 3, \pi / 3] & \mathbf{x}\left(-\pi / 3,-\pi / 3, q_{3}\right) ; q_{3} \in[-\pi / 3,0] \\
\mathbf{x}\left(\pi / 3, \pi / 3, q_{3}\right) ; q_{3} \in[0, \pi / 3] & \mathbf{x}\left(q_{1},-\pi / 3,-\pi / 3\right) ; q_{1} \in[-\pi / 3, \pi / 3]
\end{array}
$$

and $\mathbf{x}\left(q_{1}, \pi / 3, \pi / 3\right) ; q_{1} \in[0, \pi / 3]$.

Subsitituting the singular sets into Eq. (27) yields equations of curves shown in Fig. 3, which is the exact workspace of the planar 3DOF arm (Abdel-Malek and Yeh 1997). We shall use $x$ and $y$ for positioning and $\alpha$ for orienting the workspace. 


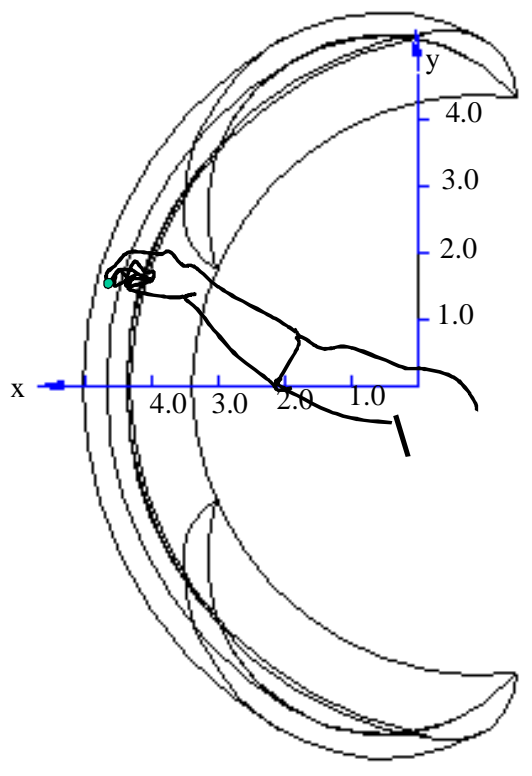

Fig. 3 The exact workspace of the arm (restricted to planar motion)

As a result of the iterative algorithm, the three design variables representing the position and orientation of the workspace are calculated as $\mathbf{w}=\left[\begin{array}{lll}x & y & \alpha\end{array}\right]^{T}$ $=\left[\begin{array}{lll}10.714 & 4.663 & 0.674\end{array}\right]^{T}$ and shown in Fig. 4.

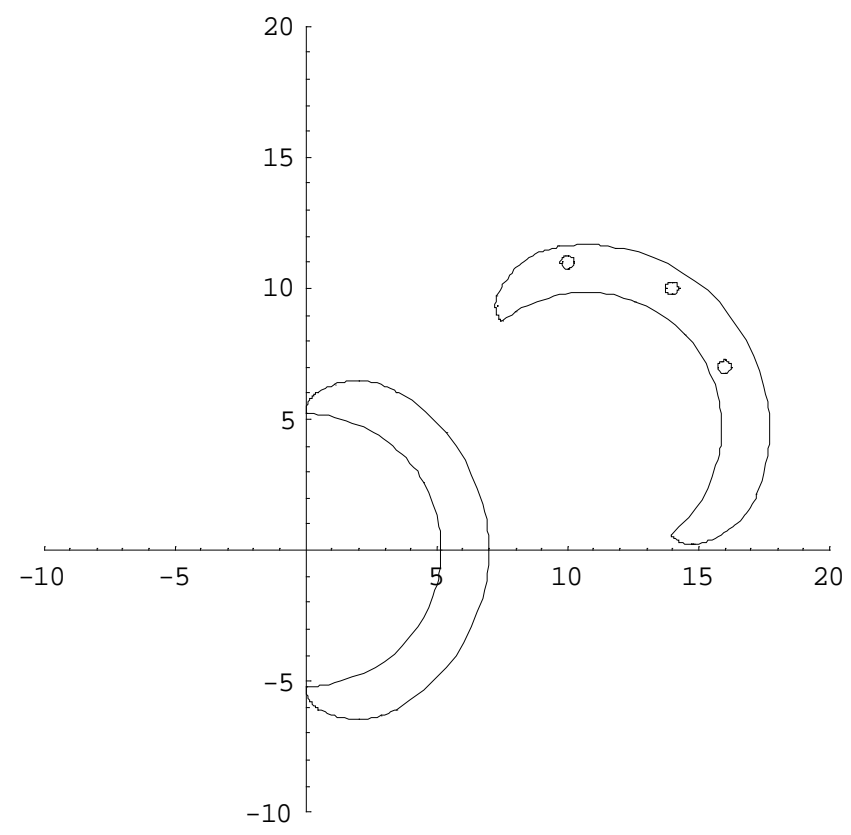

Fig. 4 The initial and final positions of the arm 
The measure of dexterity at each point is calculated as
$\mathrm{D}\left(\mathbf{P}^{(1)}\right)=79.80$
$\mathrm{D}\left(\mathbf{P}^{(2)}\right)=28.040$
$\mathrm{D}\left(\mathbf{P}^{(3)}\right)=16.914$

Note that any configuration that would have included the three points is a solution, however, the solution calculated using this method yields the position of the arm that would maximize the dexterity at all three points.

\section{Example: A spatial 3DOF arm}

Consider a 3DOF spatial manipulator where the coordinates of end-effector are given by

$$
\mathbf{x}(\mathbf{q})=\left[\begin{array}{c}
-2 \sin q_{1} \cos q_{2}+5 \cos q_{2} \\
2 \cos q_{2}+q_{3} \\
-2 \sin q_{2} \sin q_{1}+5 \sin q_{2}
\end{array}\right]
$$

where joint limits are specified by $0 \leq q_{1} \leq 2 \pi, \quad 0 \leq \mathrm{q}_{2} \leq \pi$, and $0 \leq \mathrm{q}_{3} \leq 8$.

The boundaries of the workspace consist of eight surfaces due to the singular sets listed below. The workspace is shown in Fig. 5 .

$$
\begin{aligned}
& \mathbf{s}^{(1)}=\left\{q_{1}=3 \pi / 2,0 \leq q_{2} \leq \pi, 0 \leq q_{3} \leq 8\right\} \\
& \mathbf{s}^{(2)}=\left\{q_{1}=\pi / 2,0 \leq q_{2} \leq \pi, 0 \leq q_{3} \leq 8\right\} \\
& \mathbf{s}^{(3)}=\left\{\pi \leq \mathrm{q}_{1} \leq 3 * \pi / 2,0 \leq \mathrm{q}_{2} \leq \pi, \mathrm{q}_{3}=0\right\} \\
& \mathbf{s}^{(4)}=\left\{\pi / 2 \leq \mathrm{q}_{1} \leq \pi, 0 \leq \mathrm{q}_{2} \leq \pi, \mathrm{q}_{3}=0\right\} \\
& \mathbf{s}^{(5)}=\left\{3 * \pi / 2 \leq \mathrm{q}_{1} \leq 2 * \pi, 0 \leq \mathrm{q}_{2} \leq \pi, \mathrm{q}_{3}=8\right\} \\
& \mathbf{s}^{(6)}=\left\{0 \leq \mathrm{q}_{1} \leq \pi / 2,0 \leq \mathrm{q}_{2} \leq \pi, \mathrm{q}_{3}=8\right\} \\
& \mathbf{s}^{(7)}=\left\{0 \leq \mathrm{q}_{1} \leq 2 * \pi, \mathrm{q}_{2}=0,0 \leq \mathrm{q}_{3} \leq 8\right\} \\
& \mathbf{s}^{(8)}=\left\{0 \leq \mathrm{q}_{1} \leq 2 * \pi, \mathrm{q}_{2}=\pi, 0 \leq \mathrm{q}_{3} \leq 8\right\}
\end{aligned}
$$




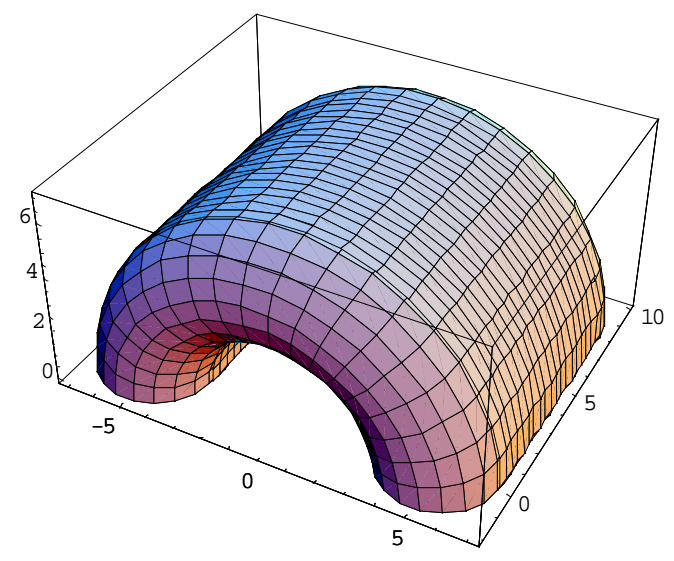

Fig. 5 The workspace of a 3-DOF manipulator

Three target points are specified as $\mathbf{p}^{(1)}(30,34,33), \quad \mathbf{p}^{(2)}(30,32,37)$, and $\mathbf{p}^{(3)}(33.4,35,35.8)$. The results of the calculations are listed in Table 2, where initial and final configurations of the manipulator are shown. The trace of the coordinates of the origin of the base is plotted in Figure 6. The relative positions of workspaces and workpoint are shown in Figure 7.

Table 2: The original and new base position

\begin{tabular}{|l|l|l|l|l|l|l|}
\hline Parameters & $\mathrm{x}_{0}$ & $\mathrm{y}_{0}$ & $\mathrm{z}_{0}$ & $\alpha$ & $\beta$ & $\gamma$ \\
\hline Initial & .000 & .000 & .000 & .000 & .000 & .000 \\
\hline Final & 34.023 & 38.594 & 37.895 & 1.762 & 4.342 & .380 \\
\hline
\end{tabular}




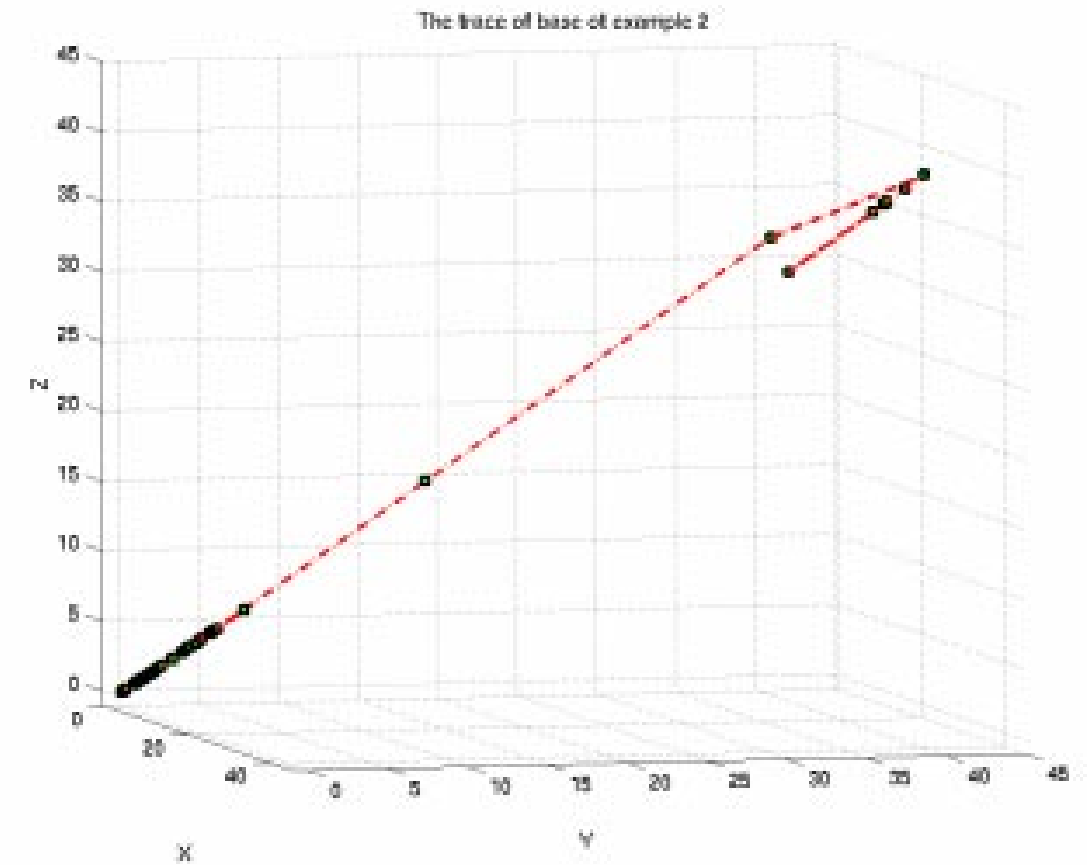

Fig. 6 The trace of the coordinates of the base of the manipulator

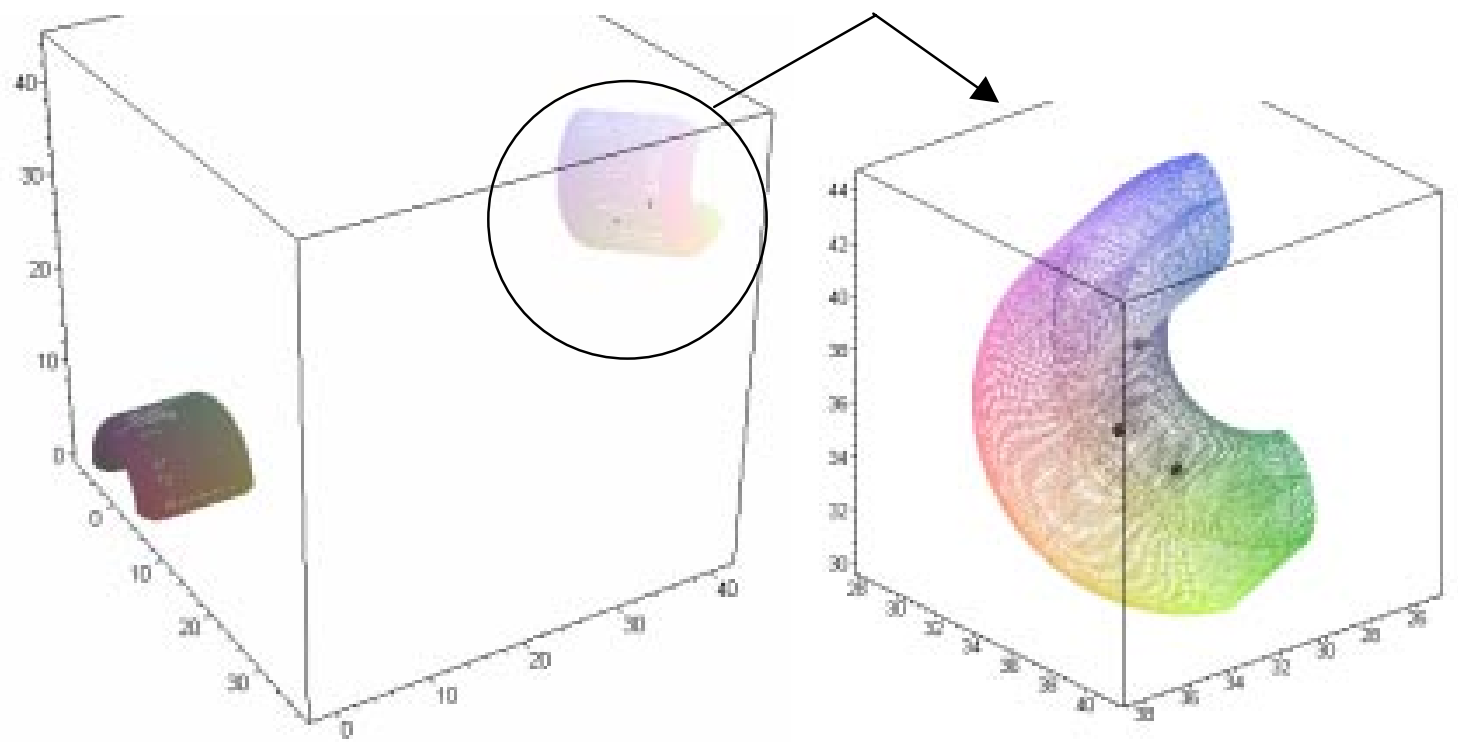

Fig. 7 Illustration of results for the placement of the 3DOF manipulator

\section{Example: A 9DOF robot manipulator}

Consider a model of the upper extremity shown in Fig. 8 and modeled as a total of 9DOF (5DOF in the shoulder, 1DOF in the elbow, and 3DOF in the wrist). Note that we have 
accounted for two translations of the shoulder joint and three rotational motions. Also note that in order to allow for realistic motion of the shoulder, we have coupled the translational and rotational joints by a linear equation such that the behavior of the motion of one joint is dependent upon the other. For the shoulder will translate by a small distance when any of the rotational joints are actuated as

$$
\begin{aligned}
& q_{3}=0.05\left(q_{1}+q_{2}\right) \\
& q_{4}=0.04\left(q_{1}+q_{2}\right)
\end{aligned}
$$

This coupling reduces the number of DOFs to seven.

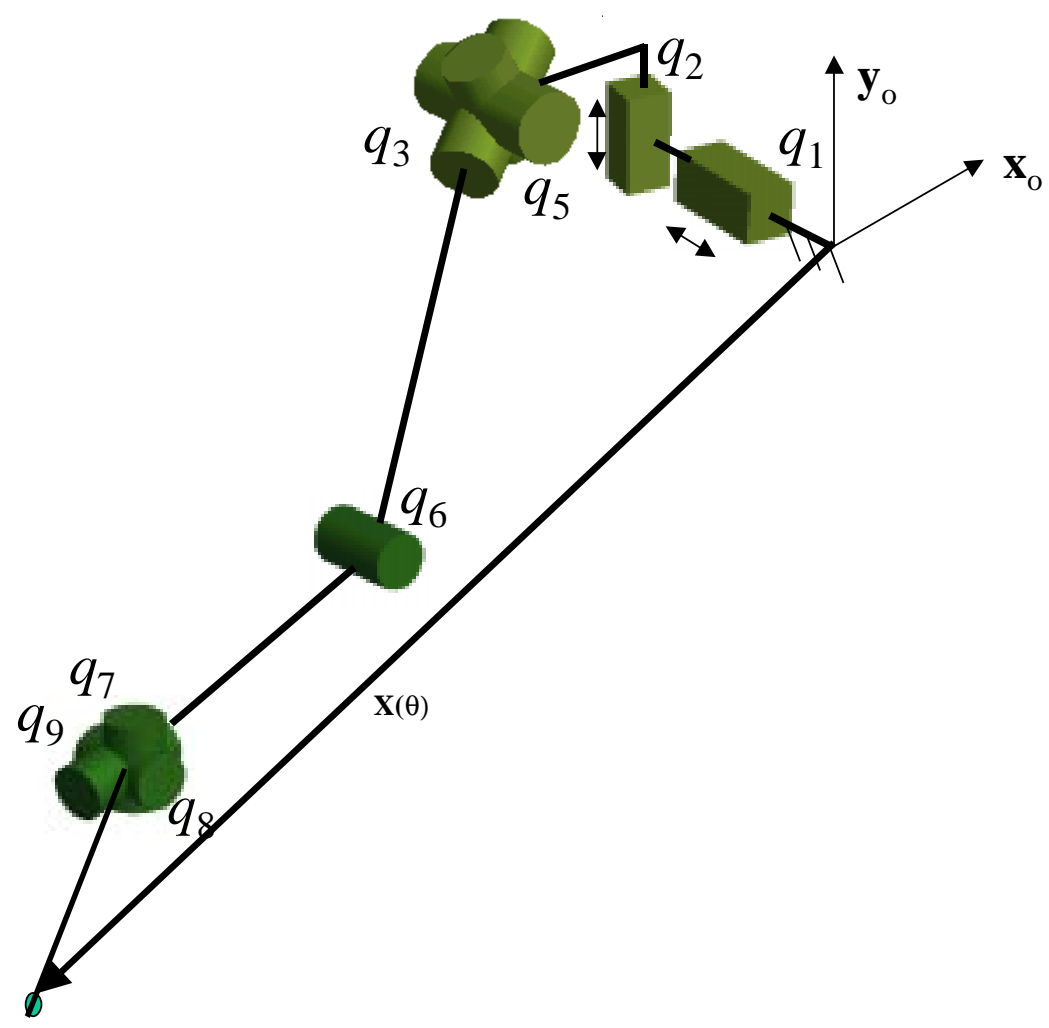

Fig. 8 A 9-DOF robot manipulator

The D-H parameters for this arm are presented in Table 2. 
Table 2: DH Table for the arm

\begin{tabular}{|l|l|l|l|l|}
\hline & $\theta_{i}$ & $d_{i}$ & $a_{i}$ & $\alpha_{i}$ \\
\hline 1 & $\pi / 2$ & $q_{1}$ & 0 & $-\pi / 2$ \\
\hline 2 & $-\pi / 2$ & $q_{2}$ & 0 & $\pi / 2$ \\
\hline 3 & $0+q_{3}$ & 0 & 0 & $\pi / 2$ \\
\hline 4 & $\pi / 2+q_{4}$ & 0 & 0 & $\pi / 2$ \\
\hline 5 & $0+q_{5}$ & 0 & $20 \mathrm{~cm}$ & $-\pi / 2$ \\
\hline 6 & $0+q_{6}$ & 0 & $25 \mathrm{~cm}$ & $\pi / 2$ \\
\hline 7 & $0+q_{7}$ & 0 & 0 & $-\pi / 2$ \\
\hline 8 & $q_{8}-\pi / 2$ & 0 & 0 & $-\pi / 2$ \\
\hline 9 & $0+q_{9}$ & 0 & 0 & 0 \\
\hline
\end{tabular}

Ranges of motion for this arm are as follows (note that the first two joints are translational): $\quad-3.8 \leq q_{1} \leq 3.8 \mathrm{~cm} ; \quad-3.8 \leq q_{2} \leq 3.8 \mathrm{~cm} ; \quad-\pi / 2 \leq q_{3} \leq \pi / 2$;

$-11 \pi / 8 \leq q_{4} \leq 2 \pi / 3 ; \quad-\pi / 2 \leq q_{5} \leq \pi / 2 ; \quad 0 \leq q_{6} \leq 5 \pi / 6 ; \quad-\pi / 3 \leq q_{7} \leq \pi / 3 ;$ $-\pi / 9 \leq q_{8} \leq \pi / 9 ;$ and $-\pi \leq q_{9} \leq 0$.

It is required to place the human (as represented by the arm's workspase) such that the following three target points are touchable and dexterity is maximized.

$$
\mathbf{P}^{(1)}=\left[\begin{array}{lll}
100 & 100 & 50
\end{array}\right]^{T}, \mathbf{P}^{(2)}=\left[\begin{array}{llll}
125 & 100 & 50
\end{array}\right]^{T}, \text { and } \mathbf{P}^{(1)}=\left[\begin{array}{lll}
125 & 125 & 75
\end{array}\right]^{T} .
$$

Note that this is a common problem that arises in the design of assembly lines, cells, and in any ergonomic design of workplaces.

The position of a point on the end-effector is determined from the Denavit-Hartenberg formulation as

$$
\begin{gathered}
x(q)=-20 c_{1} c_{3} s_{2}+20 s_{1} s_{3}+10 c_{4}\left(-c_{1} c_{3} s_{2}+s_{1} s_{3}\right)-10 c_{1} c_{2} s_{4}+5\left(c _ { 6 } \left(c _ { 5 } \left(c _ { 4 } \left(-c_{1} c_{3} s_{2}\right.\right.\right.\right. \\
\left.\left.\left.+s_{1} s_{3}\right)-c_{1} c_{2} s_{4}\right)+\left(c_{3} s_{1}+c_{1} s_{2} s_{3}\right) s_{5}\right)+\left(-c_{1} c_{2} c_{4}-\left(c_{1} c_{3} s_{2}+s_{1} s_{3}\right) s_{4}\right) s_{6}
\end{gathered}
$$




$$
\begin{aligned}
& y(\mathbf{q})=-20 c_{3} s_{1} s_{2}-20 c_{1} s_{3}+10 c_{4}\left(-c_{3} s_{1} s_{2}-c_{1} s_{3}\right)-10 c_{2} s_{1} s_{4}+5\left(c _ { 6 } \left(c _ { 5 } \left(c _ { 4 } \left(-c_{3} s_{1} s_{2}\right.\right.\right.\right. \\
&\left.\left.\left.\quad-c_{1} s_{3}\right)-c_{2} s_{1} s_{4}\right)+\left(-c_{1} c_{3}+s_{1} s_{2} s_{3}\right) s_{5}\right)+\left(-c_{2} c_{4} s_{1}-\left(-c_{3} s_{1} s_{2}-c_{1} s_{3}\right) s_{4}\right) s_{6} \\
& z(\mathbf{q})=20 c_{2} c_{3}+10 c_{2} c_{3} c_{4}-10 s_{2} s_{4}+5\left(c_{6}\left(c_{5}\left(c_{2} c_{3} c_{4}-s_{2} s_{4}\right)-c_{2} s_{3} s_{5}\right)\right. \\
&\left.+\left(-c_{4} s_{2}-c_{2} c_{3} s_{4}\right) s_{6}\right)
\end{aligned}
$$

where $c_{1}=\cos q_{1}, s_{1}=\sin q_{1}$, and $\mathbf{q}=\left[\begin{array}{lll}q_{1} & \ldots & q_{7}\end{array}\right]^{\mathbf{T}}$. Surface patches on the boundary are delineated and shown below in Fig. 9 (a total of 22 boundary surfaces):
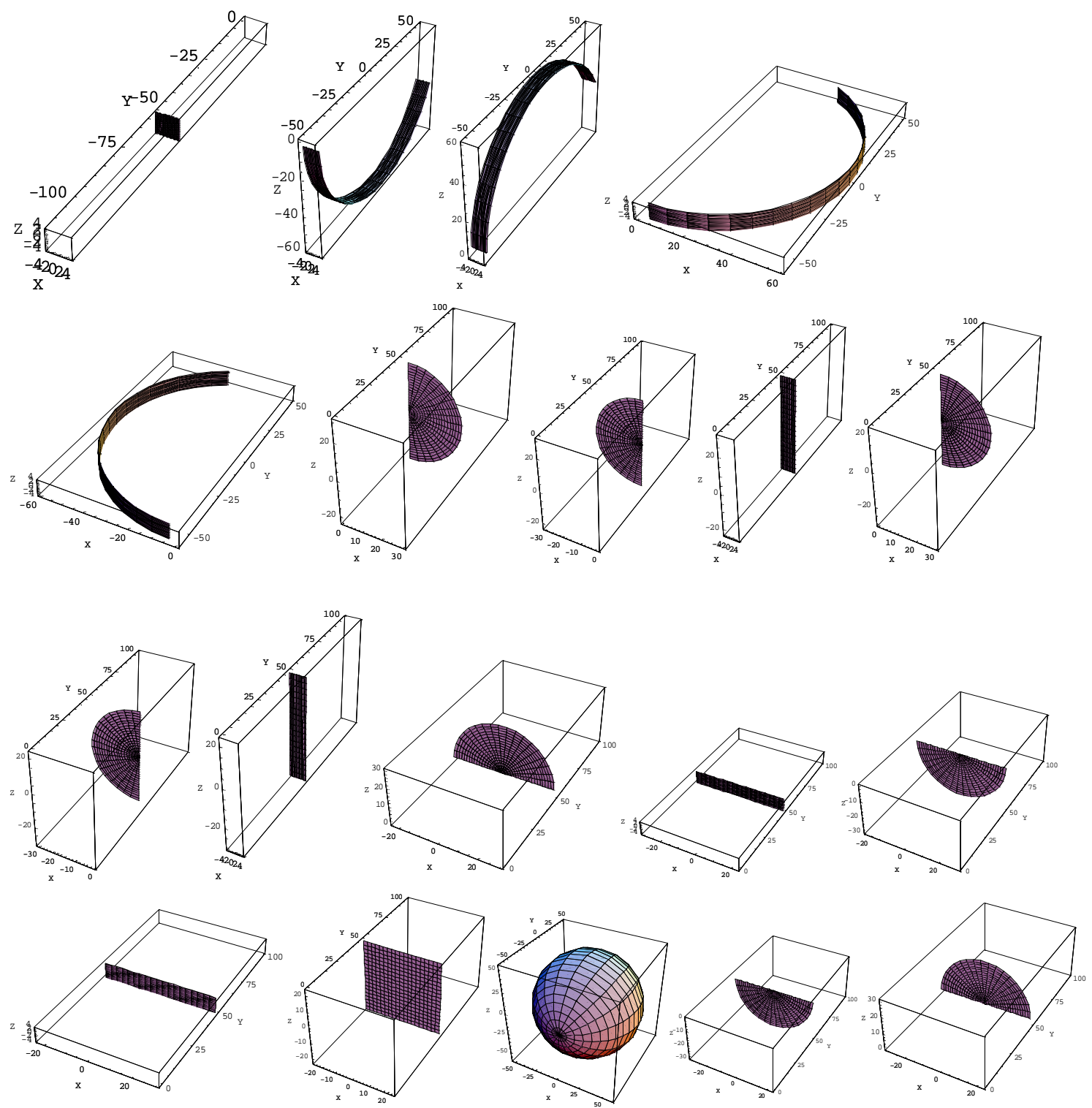

Fig. 9 Surface patches 
These surface patches are combined, the 7DOF workspace is calculated, and shown in Fig. 10. Note that we will use the six variables $\mathbf{w}=\left[\begin{array}{llllll}x_{w} & y_{w} & z_{w} & \alpha & \beta & \gamma\end{array}\right]^{T}$ to track the motion of the workspace envelope.
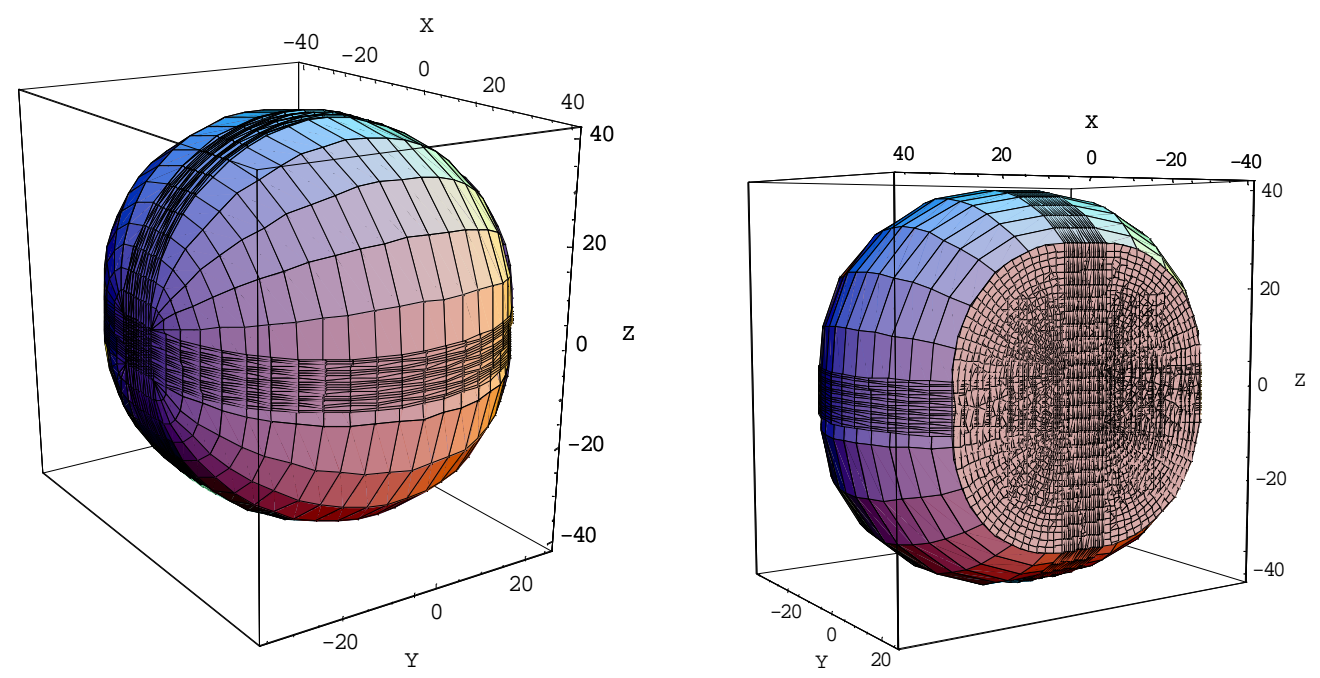

Fig. 10 Workspace of the upper extremity

As a result of the iterative algorithm, the six design variables representing the position and orientation of the workspace are calculated as

$$
\mathbf{W}=\left[\begin{array}{llllll}
107.235 & 83.444 & 94.171 & -0.850 & 1.463 & 0.736
\end{array}\right]^{T}
$$

The measure of dexterity at each point is maximized and its value is
$\mathrm{D}\left(\mathbf{P}^{(1)}\right)=11687907.83$
$\mathrm{D}\left(\mathbf{P}^{(2)}\right)=18419793.47$
$\mathrm{D}\left(\mathbf{P}^{(3)}\right)=13962690.54$

The initial and final configurations of the workspace of the arm are shown in Fig. 11. 


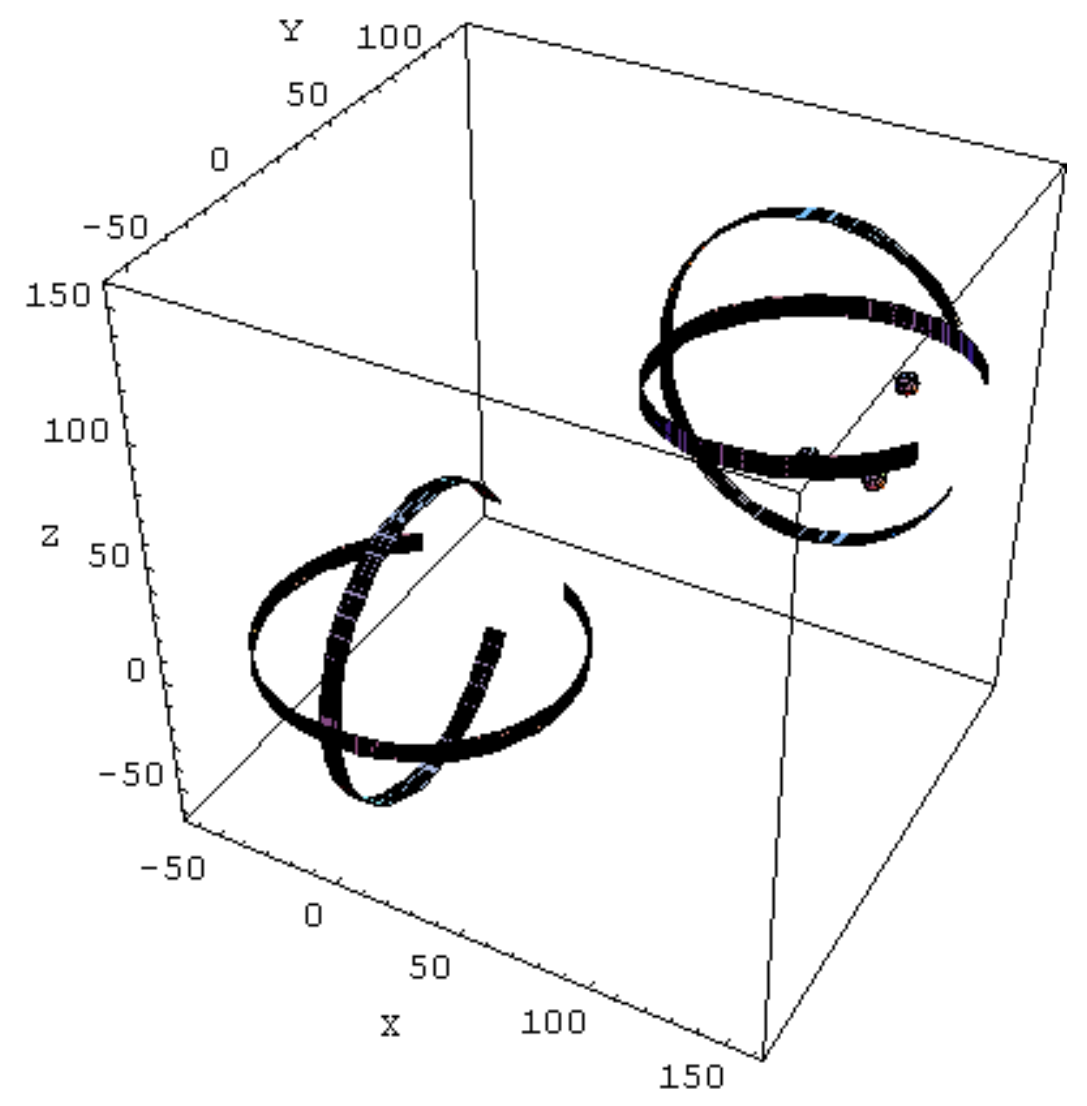

Fig. 11 Initial and final configurations of the workspace

\section{Conclusions}

A general optimization method for locating the base of a serial manipulator in a work environment while maximizing dexterity at specified target points was presented. It was shown that it is possible to place the manipulator by effecting translation and orientation of the workspace generated in closed form and characterized by surface patches on the boundary. It was shown that the placement problem can be formulated as an optimization problem where the cost function is dexterity and the constraints pertain to including the target points in the workspace. 
A new dexterity measure was introduced that takes into account singular behavior and joint limits, which is a fundamental improvement over that reported by Yoshikawa (1995).

It was shown that the proposed dexterity measure can be used as a cost function in an optimization algorithm whereby the robot workspace's motion is tracked using six generalized variables. The final position and orientation of these variables determine the placement of the base. The method and code were demonstrated using a simple planar example and using a spatial model of the upper extremity with 9DOFs.

\section{References}

Abdel-Malek, K. and Yeh, H.J., (1997), "Path Trajectory Verification for Robot Manipulators in a Manufacturing Environment" IMechE Journal of Engineering Manufacture, Vol. 211, part B, pp. 547-556.

Abdel-Malek, K., Adkins, F., Yeh, H.J., and Haug, E.J. (1997), "On the Determination of Boundaries to Manipulator Workspaces," Robotics and Computer-Integrated Manufacturing, Vol. 13, No. 1, pp.63-72.

Abdel-Malek, K., Yeh, H-J, and Khairallah, N., (1999), "Workspace, Void, and Volume Determination of the General 5DOF Manipulator, Mechanics of Structures and Machines, 27(1), 91-117.

Bergerman, M; Xu, Y, 1997, "Dexterity of underactuated manipulators", Proceedings of the 1997 8th International Conference on Advanced Robotics, ICAR'97 Jul 7-9, 1997, Monterey, CA, pp. 719-724.

Bicchi, A.; Melchiorri, C., 1993, "Manipulability measures of cooperating arms", Proceedings of the 1993 American Control Conference Jun 2-4, IFAC, pp. 321-325.

Denavit, J. and Hartenberg, R.S., (1955), "A Kinematic Notation for Lower-Pair Mechanisms Based on Matrices", Journal of Applied Mechanics, vol.77, pp.215221.

Kim, Jin-Oh; Khosla, Pradeep, K., 1991, "Dexterity measures for design and control of manipulators", Proceedings of the IEEE/RSJ International Workshop on Intelligent Robot and Systems '91, v 2, pp. 758-763.

Lee, J., 1997, "Study on the manipulability measures for robot manipulators", Proceedings of the 1997 IEEE/RSJ International Conference on Intelligent Robot and Systems. Part 3 (of 3) Sep 7-11 1997 v 31997 Grenoble, France, pp. 14581465. 
Pamanes-Garcia, J.A., 1989, "A Criterion for the Optimal Placement of Robotic Manipulators", Proceedings IFAC Information Control Problems in manufacturing Technology, Madrid, Spain.

Park, F.C.; Brockett, R.W., "Kinematic dexterity of robotic mechanisms", International Journal of Robotics Research, v 13 n 1 Feb 1994 p 1-15.

Rastegar, J.; Singh, J.R., 1994, "New probabilistic method for the performance evaluation of manipulators", ASME Journal of Mechanical Design, v 116 n 2, pp. 462-466.

Seraji, H., 1995, "Reachability Analysis for Base Placement in Mobile Manipulators", Journal of Robotic Systems, Vol. 12(1), pp. 29-43.

Sturges, R.H., 1990, "Quantification of machine dexterity applied to an assembly task", International Journal of Robotics Research, v 9 n 3 Jun 1990 pp. 49-62.

Wang, J.Y.; Wu, J.K., 1992, "Computational environment for dextrous workspace analysis", DE Advances in Design Automation-Proceedings of 18th Annual Design Automation Conference, v 44 pt 2, pp. 293-302.

Yang, F.-C.; Haug, E. J., 1991, "Numerical analysis of the kinematic working capability of mechanisms", DE Advances in Design Automation-Proceedings of 17th Design Automation Conference, v 32 pt 1, pp. 9-17.

Yoshiakawa, T., 1985, Manipulability of Robotic Mechanisms", International Journal of Robotics Research, Vol. 4(2), pp. 3-9.

Youheng, X; Kaidong, Z, 1993, "Optimum synthesis for workspace dexterity of manipulators", Journal of Shanghai Jiaotong University, v 27 n 41993 p 40-48.

Zeghloul, S., Pamanes-Garcia, J.A., 1993, "Multi-criteria optimal placement of robots in constrained environments", Robotica, Vol. 11, pp. 105-110.

\section{Appendix A}

Using the Denavit-Hartenberg representation, the position of the end-effector can be

analytically formulated as

$$
\mathbf{x}=\boldsymbol{\Phi}(\mathbf{q})
$$

where $\mathbf{q}=\left[q_{1}, q_{2}, \ldots, q_{n}\right] \in \mathbf{R}^{n}$ is the vector of joint coordinates. Joint limit constraints are imposed using the transformation defined in Eq. (7) as $\mathbf{q}=\mathbf{q}(\boldsymbol{\lambda})$. For any admissible configuration, the following $(n+3)$ augmented constraint equations must be satisfied

$$
\mathbf{G}(\mathbf{z})=\left[\begin{array}{l}
\Phi(\mathbf{q})-\mathbf{x}_{o} \\
\mathbf{q}(\lambda)-\mathbf{q}_{o}
\end{array}\right]=\mathbf{0}
$$

where the augmented vector $\mathbf{z}=\left[\mathbf{x}^{T}, \mathbf{q}^{T}, \lambda^{T}\right]^{T}$. Singularity sets resulting from a row-rank deficiency criteria must be determined to generate envelopes to the workspace. These 
envelopes are characterized by surface patches that exist inside, outside, and extending both internal and external to the workspace. The input Jacobian of $\mathbf{G}\left(\mathbf{q}^{*}\right)$ is obtained by differentiating $\mathbf{G}$ with respect to $\mathbf{z}$ as

$$
\mathbf{G}_{\mathbf{z}^{*}}=\left[\begin{array}{cc}
\Phi_{\mathbf{q}} & \mathbf{0} \\
\mathbf{I} & \mathbf{q}_{\lambda}
\end{array}\right]
$$

which is an $(n+3) \times(2 n)$ matrix, where $\Phi_{\mathbf{q}}=\partial \Phi_{i} / \partial q_{j}$ is a $(3 \times n)$ matrix, $\mathbf{I}$ is the $(n \times n)$ identify matrix, and $\mathbf{q}_{\lambda}=\partial q_{i} / \partial \lambda_{j}$ is an $(n \times n)$ diagonal matrix with diagonal elements as $\left.q_{\lambda}\right)_{i i}=b_{i} \cos \lambda_{i}$, where $\mathbf{G}_{\mathbf{z}}$ is defined as the augmented Jacobian matrix.

The objective is to find the constant subvectors of $\mathbf{q}$, denoted by $\mathbf{s}$, which make the subJacobian $\mathbf{G}_{\mathbf{z}}$ row rank deficient. Three singularity types are identified:

(1) Jacobian singularities (called Type I) that satisfy

$$
S^{(1)} \equiv\left\{\mathbf{s} \in \mathbf{q}: \operatorname{Rank}\left[\Phi_{\mathbf{q}}\right]<\mathbf{3}, \text { for some constant } \mathbf{s}\right\}
$$

(2) A set characterized by a null space criteria imposed on the reduced-order manipulator (called Type II singular set)

$$
S^{(2)}=\left\{\mathbf{s} \in \mathbf{q} ; \operatorname{dim}\left[\operatorname{Null}\left[\Phi_{\mathbf{q}}^{T}(\mathbf{q})\right]\right] \geq 1, \text { for some } \mathbf{q}\right\}
$$

where $\Phi_{\mathbf{q}}(\mathbf{q})$ is the Jacobian after reducing the order of the manipulator.

(3) The set defined by a combination of all constant generalized coordinates (called Type III singular set)

$$
\left.S^{(3)}=\left\{\mathbf{s} \in \mathbf{q} ; \llbracket q_{i}^{o} \quad q_{j}^{o}\right], \text { for } i, j=1 \text { to } n ; i \neq j\right\}
$$

Substituting these singular sets into the position vector defined by Eq. (a1) yields parametric singular geometric entities (curves or surfaces) defined by 


$$
\xi^{(i)}\left(\mathbf{u}^{(i)}\right) \equiv \boldsymbol{\Phi}\left(\mathbf{u}^{(i)}, \mathbf{s}^{(i)}\right)
$$

Intersections between these singular surfaces may exist. Moreover, these curves partition a singular surface into a number of regions called subsurfaces. The result is the identification of all boundary surface patches that characterize the manipulator's workspace. 